\title{
Stop \& Go ABR: A Simple Algorithm for the Implementation of Best Effort Services in ATM LANs
}

\author{
M. Ajmone Marsan ${ }^{1}, K$. Begain ${ }^{2}$, R. Lo Cigno ${ }^{1}$, M. Munafó ${ }^{1}$
}

${ }^{1}$ Dipartimento di Elettronica, Politecnico di Torino

Corso Duca degli Abruzzi, 24 - 10129 Torino - Italy

e-mail: $\{$ ajmone,locigno,munafo\}@polito.it

${ }^{2}$ Department of Computer Science, Mu'tah University

Mu'tah 61710 - Jordan e-mail: begain@science.sci.mutah.edu.jo

\begin{abstract}
In this paper we propose a novel algorithm for the implementation of best effort services in ATM LANs. The algorithm is a peculiar version of ABR in which sources can transmit only at two different cell rates, the Peak Cell Rate (PCR) and Minimum Cell Rate (MCR); for this reason, the proposed algorithm is called Stop \& Go ABR. The Stop \& Go ABR algorithm is first described, and then evaluated by detailed simulation of a simple ATM LAN configuration with best effort TCP connections as well as interfering VBR connections, showing that it is capable of providing very good performance and fairness.
\end{abstract}

\section{Keywords}

ATM, traffic management, ABR, performance

\section{INTRODUCTION}

The data communication services typical of computer networks, such as Local Area Networks (LANs), Metropolitan Area Networks (MANs), and, most important, the Internet, are based on a datagram, or connectionless, packet switching approach, and generally do not guarantee the success in the delivery of the information, thus being often termed Best-Effort.

This is in contrast with the traditional approaches adopted in telecommunication networks, that are based on either circuit switching or packet switching with virtual circuits, also called connections, and that try to guarantee the delivery at the destination of the information generated at the source.

The original conception of the Asynchronous Transfer Mode (ATM) was due to researchers in the telecommunications field, and as a result ATM is based on connection-oriented cell switching, and is naturally matched to guaranteed services, not to best effort services.

The selection of the most adequate approach for the provision of best effort 
services in ATM networks has been the subject of many debates within the technical literature as well as standardization committees.

Today, two techniques are standardized for the provision of best effort services in ATM networks, known as the UBR and ABR service categories or transfer capabilities [6, 5].

UBR stands for Unspecified Bit Rate; UBR provides very simple means for the transfer of the data resulting from best effort services through ATM networks. The problem of UBR is that it can be quite inefficient $[13,11,2]$, depending on the network configuration and load.

ABR stands for Available Bit Rate; ABR provides algorithms with variable degree of sophistication and efficiency to exploit the bandwidth not used by guaranteed services for the transfer of the data resulting from best effort services through ATM networks. The problem of ABR is that the algorithms that permit good performance to be obtained are rather complex to implement.

In particular, three ABR operating modes are specified (named EFCI, RRM and ERM respectively, see [5] for their description); all are based on the use of special flow control cells, called Resource Management (RM) cells, to notify sources about the congestion of ATM switches along the path followed by the connection. ABR sources are required to react to the information arriving from the network by adequately modifying (increasing or reducing) their cell transmission rates.

The key to success of an ABR implementation is threefold: i) high performance; ii) robustness and great resilience; iii) low implementation cost both for the end-user and for the network nodes. Several proposals have appeared in literature, based either on theoretical approaches $[10,15,9]$, or on more heuristic and empiric considerations [7, 12,3]. Most of them try to optimize performance, while robustness and cost received less attention.

In this paper we propose a novel algorithm for the implementation of best effort services in ATM LANs that is a peculiar version of ABR in which sources can transmit only at two different cell rates, the Peak Cell Rate (PCR) and Minimum Cell Rate (MCR). For this reason, the proposed algorithm is called Stop \& Go ABR.

\section{STOP \& GO ABR}

Stop \& Go ABR is a version of $A B R$ that was designed with the following main objectives in mind: i) be extremely simple to implement, both at end user ATM terminals and within ATM switches; ii) be acceptably efficient and fair in typical LAN environments; iii) be compliant with the ATM Forum Traffic Management Specification [5].

Stop \& Go ABR can be considered a particular version of either the Relative Rate Marking (RRM) or the Explicit Rate (ER) ABR operating modes, that allows only two values for the transmission speed of ABR sources: the Minimum Cell Rate (MCR) and the Peak Cell Rate (PCR). 


\begin{tabular}{lclclc}
\hline Parameter & Value & Parameter & Value & Parameter & Value \\
\hline Nrm & 32 & Mrm & 2 & TCR & 10 \\
\hline RIF & 1 & RDF & 1 & CDF & 1 \\
\hline TBE & ID & ATDF & ID & Trm & ID \\
\hline MCR & ID & PCR & ID & ICR & PCR/MCR \\
\hline
\end{tabular}

Table 1 Parameter vector for Stop \& Go ABR implementation, the label I.D. means that values are implementation dependent

In this paper we shall consider Stop \& Go ABR as deriving from RRM ABR by setting both the Rate Increase Factor (RIF) and the Rate Decrease Factor (RDF) to 1 .

Table 1 gives the ABR parameter vector that must be used in order to implement Stop \& Go ABR. Most parameter values are fixed, hence reducing the complexity of the negotiation at connection setup; the others, identified in the table as Implementation Dependent (ID), can be either negotiated at connection setup or set by the network manager to a suitable value.

For what the Initial Cell Rate (ICR) is concerned, it is assumed that ICR = PCR; however, if the network is not able to support a value of the Transient Buffer Exposure (TBE) large enough to ensure that $\frac{\text { TBE }}{\text { FRTT }} \geq$ PCR where FRTT is the round trip time measured at connection setup, then ICR must be set to MCR.

The behavior of the source and destination ATM equipment in Stop \& Go ABR is exactly as specified in Sections 5.10.4 and 5.10.5 in [5].

The switch behaviors are not standardized in [5], in order to leave space for the competition among equipment manufacturers. The study of Stop \& Go ABR in this paper assumes that ATM switches use two separate buffers for each output interface: one buffer is used for high priority connections (CBR and/or VBR) and the second for ABR connections (that are considered to be of lower priority); moreover, switches are assumed to implement RRM ABR with a control algorithm based only on the ABR buffer occupancy.

More precisely, the control algorithm within ATM switches is based on the comparison of the ABR queue length $Q_{l}$ with a threshold $t$. The NI (No Increase) and CI (Congestion Indication) bits within backward RM cells are set as follows for all ABR connections:

$$
\begin{array}{lll}
Q_{l}<t & \Rightarrow \mathrm{NI}=0 & \mathrm{CI}=0 \\
Q_{l} \geq t & \Rightarrow \mathrm{NI}=1 & \mathrm{CI}=1
\end{array}
$$

with no specific algorithm for the enforcement of fairness among different connections; in practice, the NI bit is not used. 
In general, in RRM ABR, the NI and CI bits are used to form a 3-state feedback (when $\mathrm{CI}=1$, NI has no meaning) with the semantic "increase the cell rate" $(\mathrm{NI}=\mathrm{CI}=0)$, "keep the actual cell rate" $(\mathrm{NI}=1, \mathrm{CI}=0)$, and "decrease the cell rate" $(\mathrm{NI}=-, \mathrm{CI}=1)$, but with just one threshold in the buffer it is impossible to discriminate more than two cases.

The use of two thresholds in order to create an hysteresis cycle, and also use the NI bit, was shortly investigated, but was discarded for the following two reasons: i) a "Stop \& Go" system has intrinsically only two states, so that the $(\mathrm{NI}=1, \mathrm{CI}=0)$ combination has little meaning; ii) traffic configurations may arise where $Q_{l}$ remains between the two thresholds while some connections are transmitting at their PCR and others are transmitting at their MCR, thus creating stable, unfair situations.

Different ATM switch control algorithms, derived either from RRM ABR or from ER ABR, are being considered for further study on Stop \& Go ABR.

\section{PERFORMANCE EVALUATION WITH TCP CONNECTIONS}

Stop \& Go ABR was designed for the implementation of best effort services in LAN environments, where round trip delays are short, and congestion is not likely to arise in more than one node at a time.

The performance analysis of Stop \& Go ABR in such a setup must take into account the interactions between the control algorithms of Stop \& Go ABR and those of TCP, that is by far the most widely used transport protocol for the provision of best-effort services in LANs.

For this reason, in the characterization of the performance of Stop \& Go ABR we studied its behavior with TCP connections in a rather simple LAN setup. We restrict the analysis to greedy TCP connections transmitting only maximum size segments whose dimension is 1460 bytes (this value is the one presently used on Ethernet LANs) excluding all overheads; to those we add a 48-byte overhead ( 20 bytes for the TCP header, 20 for the IP header, and 8 for AAL5 overheads). The TCP implementation used in the simulations is the officially distributed BSD TCP-reno 4.3, that was adapted to run on top of CLASS* [1, 4], a cell level ATM network simulator developed at Politecnico di Torino in co-operation with CSELT, the research centre of Telecom Italia. The only major modification introduced into the TCP code concerns the timer granularity that was set to $50 \mu \mathrm{s}$, in order to adapt it to large bandwidth-delay product networks.

*CLASS stands for Cell Level ATM network Services Simulator; it is entirely written in $\mathrm{C}$ language and portable on most computing platforms. More information on CLASS are available at the URL http://घrr.tlc.polito.it/class.html. 


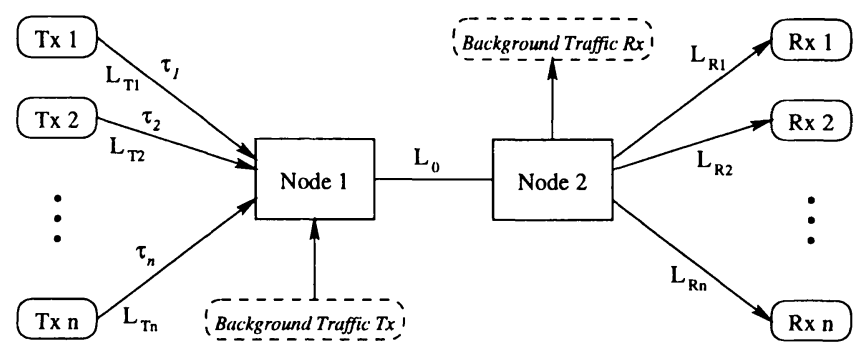

Figure 1 Bottleneck topology

\begin{tabular}{lclclc}
\hline Parameter & Value & Parameter & Value & Parameter & Value \\
\hline TBE & 500 & ATDF & 10.0 & Trm & 100 \\
\hline MCR & $1 \mathrm{Mbit} / \mathrm{s}$ & ICR & PCR & & \\
\hline
\end{tabular}

Table 2 Implementation dependent ABR parameter values used in the simulations

\subsection{The simulation scenario}

Fig. 1 presents the "bottleneck" topology, that can be considered a simplified representation of any LAN scenario, where only the congested node (node 1) is considered, together with the sources and destinations that load the congested output link.

Several TCP transmitters (say $n$ ) are connected to node 1 , and the corresponding TCP receivers are connected to node 2 . The $n$ TCP transmitters contend for the transmission resources on the congested link connecting nodes 1 and 2. Both nodes implement Stop \& Go ABR as described in Sect. 2; however, only node 1 is congested, due to the demultiplexing in node 2 of all the TCP connections that cross the only link connecting the two nodes. The same congested link is also used by some background traffic that is not controlled with the ABR mechanism, since it is supposed to be generated by CBR or VBR sources, hence to have higher priority. TCP connections are unidirectional, and TCP receivers return only ACKs to the corresponding transmitters.

Table 2 reports the values of the implementation dependent Stop \& Go ABR parameters used in the simulations. The PCR value is one of the variables of our study. The other variables in the study are the number of TCP connections, the background traffic characteristics, and the network span.

In the scenario taken as a reference for the simulation experiments we assume that all links in the network have capacity $150 \mathrm{Mbit} / \mathrm{s}$, that the link between the two nodes is $10 \mathrm{~km}$ long, and that the distance of transmitters from node 1 and that of receivers from node 2 are also $10 \mathrm{~km}$, that is, 
$L_{0}=L_{T i}=L_{R i}=10 \mathrm{~km}$ in Fig. 1. Such distances correspond to a round trip delay approximately equal to $0.15 \mathrm{~ms}$, which can be assumed to be typical for medium size LANs, if processing delays are taken into account. The number of TCP connections is set to 3 , and the background traffic is assumed to be generated by one On-Off VBR source with exponentially distributed On and Off periods that transmits at constant bit rate $C_{b}$ during On periods. The average durations of the On and Off periods are 20,000 and 10,000 slots, respectively (roughly 56 and $28 \mathrm{~ms}$ ), while $C_{b}$ varies with the background traffic load; namely $C_{b}$ is 1.5 times the value of the background traffic load values in the horizontal axis of the plots shown in the following Subsection. The buffer sizes within the switch are 100 cells for the higher priority traffic (that does not need a large buffer), and 1,000 cells for the lower priority ABR traffic. This latter value corresponds to a buffer size of $53 \mathrm{kbytes}$, that is fairly small for the equipments available on the market. The threshold is set to $25 \%$ of the ABR buffer size; namely $t=250$.

If not otherwise stated, numerical results are obtained with the values just described for the reference simulation scenario.

In order to measure the network performance, we use three different metrics.

TCP goodput - this is the throughput seen by the TCP end user, after all faulty and duplicated TCP segments have been discarded.

TCP efficiency - this is the ratio between the goodput and the load offered by the TCP transmitter.

Link Waste (LW) - this is the bandwidth that remains unused on the bottleneck link, expressed as a fraction of the link capacity.

\subsection{Numerical results}

Let us first of all analyse the impact of the PCR value on the network performance in the reference simulation scenario with Stop \& Go ABR.

Fig. 2 presents 4 plots where the value of the PCR of the three TCP connections is varied.

All results are plotted against the background offered load. The left vertical scale measures the goodput of the three TCP connection, that are reported with solid lines; the dot-dashed line represent the capacity theoretically available for each TCP connection (thus, ideally, all solid lines should lie on the dot-dashed line). The left vertical scale measures the efficiency of the three TCP connections (reported with dashed lines, that ideally should be equal to 1 ), as well as the link waste (reported as a dotted line, that hopefully should be as near as possible to 0 ).

The 4 plots refer to the values PCR $=25,50,75$, and $100 \mathrm{Mbit} / \mathrm{s}$, as indicated on each plot. The overall network performance is quite satisfactory, showing a good exploitation of the network resources with high efficiency, 

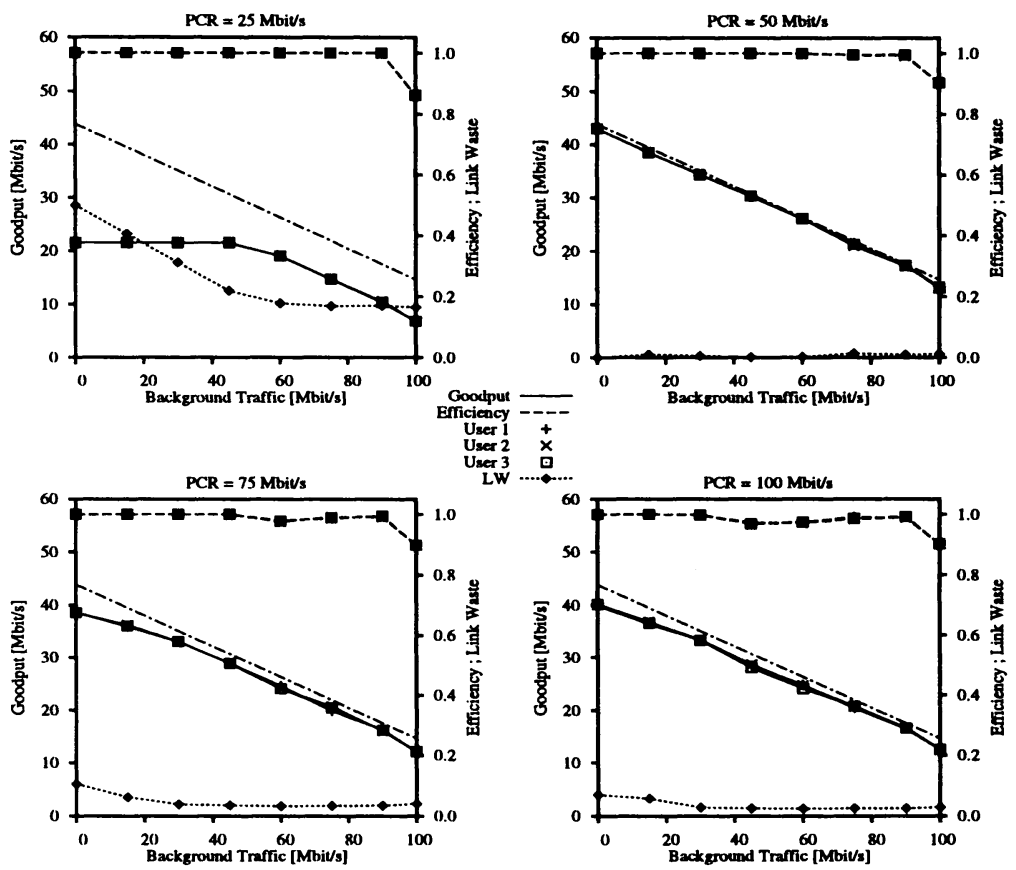

Figure 2 Performance with 3 TCP connections with equal length and PCR

together with a surprising degree of fairness, given the simplicity of the implementation.

It is remarkable that also when the peak load offered by the ABR connections $(\mathrm{PCR}=100 \mathrm{Mbit} / \mathrm{s}$ - lower right plot) is twice the link capacity, the behavior of the network is fair and quite smooth, while it has been shown $[13,11,8,2]$ that plain TCP over ATM generally shows a poor behavior.

Considering the plot for $\mathrm{PCR}=25 \mathrm{Mbit} / \mathrm{s}$, one might expect that, as the background load increases and reaches $75 \mathrm{Mbit} / \mathrm{s}$, the goodput of the TCP connections, that at lower loads were rate limited, reaches the available capacity. In fact, the characteristics of the background traffic prevent this behavior: since the background traffic is generated by one On-Off source, the spare capacity mainly results from silence periods of the On-Off source, that the TCP connections can not exploit due to their PCR limitation.

One final remark on the $\mathrm{PCR}=50 \mathrm{Mbit} / \mathrm{s}$ plot: the performance in this case is so much better than in other cases because the sum of the PCRs is exactly equal to the link capacity and the background traffic is On-Off - a situation that is probably not realistic.

A specific comment is needed for the efficiency curves. It is in general expected that the efficiency drops below 1 when cells are lost in the network and, as a consequence, faulty segments are discarded at the receiver, triggering the retransmission of the lost segment either for a timeout expiration or, more probably, for repeated ACKs reception at the source. However, no 
TCP cells are dropped either from the ABR buffer in the congested node, or anywhere else in the network during the simulation runs, hence the efficiency reduction is due to some different cause. As a matter of fact, the expiration of a retransmission timer is not necessarily triggered by a segment loss, but it can be due to an exceedingly long time before the ACK comes back. The TCP retransmission timer $\tau_{o}$ are set according to the formula $\tau_{o}=\hat{\mu}_{t}+4 \hat{\sigma}_{t}$ where $\hat{\mu}_{t}$ and $\hat{\sigma}_{t}$ are smoothed estimations of the round trip time average and standard deviation computed by TCP with the algorithm proposed by Van Jacobson (see [14] pp. 300). When the background is Off for a reasonably long time $\hat{\mu}_{t}$ is very close to the propagation round trip time, i.e., $\simeq 0.15 \mathrm{~ms}$ in our case, and $\hat{\sigma}_{t}$ is very small, so that $\tau_{o}$ is set to a very small value, say $<1 \mathrm{~ms}$. When the background traffic source switches to the On state, it is served with priority within the node, and TCP traffic must wait until capacity is available to serve it. The PCR of the background traffic for a background load of $100 \mathrm{Mbit} / \mathrm{s}$ is $150 \mathrm{Mbit} / \mathrm{s}$, so that during the whole duration of an On period, whose average is roughly $56 \mathrm{~ms}$, TCP traffic is not served: under these circumstances it is common that a timeout expires even if no cells have been lost in the network. It might be argued that setting the TCP timer granularity to a larger value can avoid this phenomenon, but indeed this is true only to a very limited extent. If the granularity is increased to 100 or $500 \mu \mathrm{s}$, the qualitative behavior described above is not modified; if the timer granularity is instead increased to a value much larger than the propagation delay, say 50 or $100 \mathrm{~ms}$, that are values pretty similar to those used in today implementations, the above phenomenon is probably avoided, but TCP becomes completely unable to adapt to network changes since its sensitivity is too low to detect congestion.

The efficiency reduction is due to this phenomenon not only in the results presented in Fig. 2, but also in all other results presented throughout the paper, since cell losses were never observed in simulation runs.

Let us now consider a slightly different situation, where each TCP source is at a different distance from the congested ATM switch.

Fig. 3 reports the results for the same PCR values of Fig. 2, but with link lengths $L_{1}=1 \mathrm{~km}, L_{2}=5 \mathrm{~km}$ and $L_{3}=10 \mathrm{~km}$, respectively.

The behavior of TCP is known to be biased against longer connections; however, if the ABR mechanism is able to prevent cell losses, the TCP congestion control mechanism is not triggered, and the performance is dictated by the ABR control loop, whose behavior is influenced by the loop delay. Fig. 3, however, shows that the overall network performance is satisfactory, and even fairness does not suffer appreciably form the different source distances from the control point, even if they vary over an order of magnitude. The reason for this quite good behavior lies in the fact that the ABR feedback is intrinsically sampled: neglecting delay jitters, it is sent once every $t_{f}=\frac{1}{\mathrm{Nrm}} \cdot R_{e} \cdot t_{s}$ seconds, 

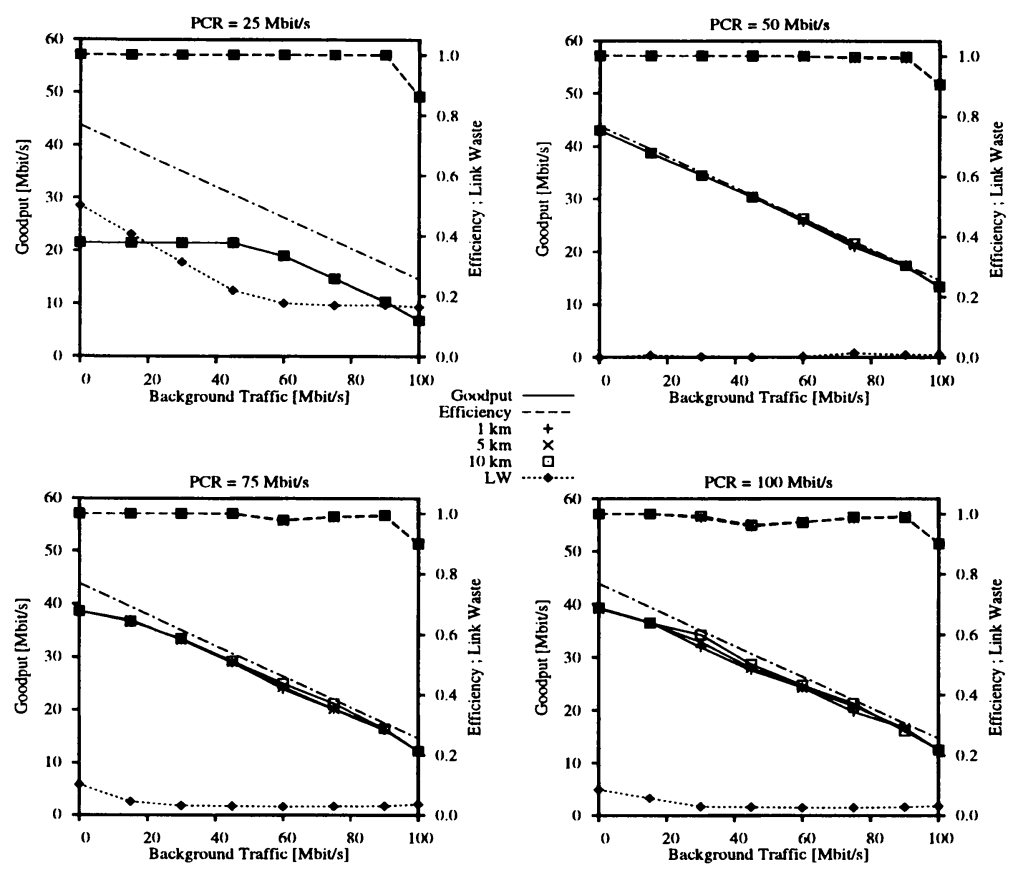

$\begin{array}{ll}\mathrm{km} & + \\ \mathrm{km} & \times \\ \mathrm{km} & \square\end{array}$

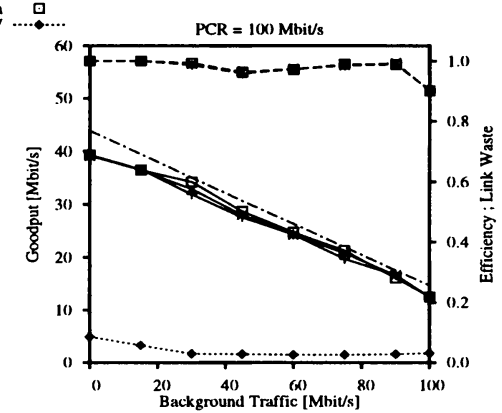

Figure 3 Performance with 3 TCP connections with different length and equal PCR

where $R_{e}$ is the effective rate of the source*, and $t_{s}$ is the slot duration. In the case under consideration $t_{f}$ varies between $136 \mu \mathrm{s}$ when $R_{e}=100 \mathrm{Mbit} / \mathrm{s}$ and $13.6 \mathrm{~ms}$, when $R_{e}=1 \mathrm{Mbit} / \mathrm{s}$ : both values are larger than the control loop delay of the $10 \mathrm{~km}$ connection that is roughly $50 \mu \mathrm{s}$. In practice this means that the sampled closed loop system has a transfer functions whose response delay is equal to 1 sample independently from the source distance.

Fig. 4 shows performance results in the case when TCP connections have different PCRs; both cases of equal length connections and of different length connections are considered.

The goodput of TCP connections is proportional to their PCR, as it might be expected, and as it is probably perceived as "fair" from both the users and the network point of view. Once again, connection lengths have a marginal impact on performance; in particular, if we observe the lower plots, that refer to the case of different length connections, it is clear that the source with higher PCR gets the most out of the network, independently from being the nearest or the farthest from the congested node.

Up to now we have considered a rather small LAN scenario, where Stop \& Go ABR proved to perform quite well; this scenario was considered because

*If the connection is persistent, as in our study, $R_{e}$ is equal to the Current Cell Rate (CCR) read in the backward RM cells; however in the general case the source can transmit at lower rates, or even be silent. 

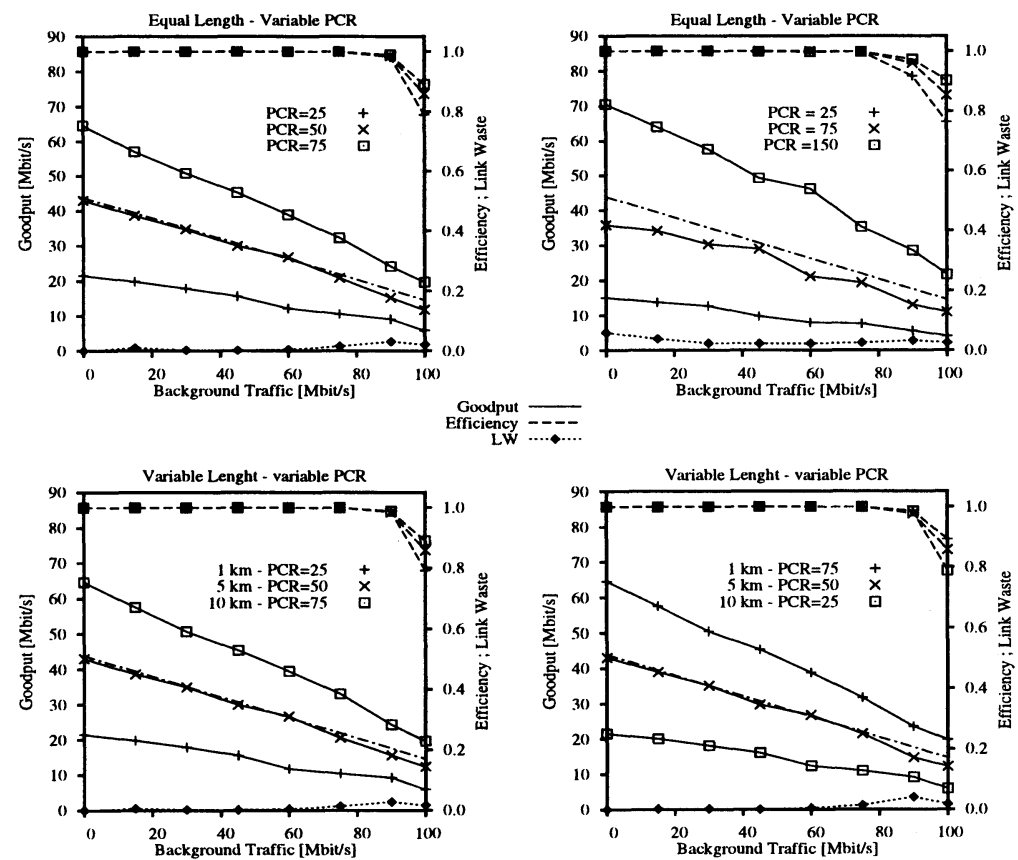

Figure 4 Performance with 3 TCP connections with different lengths and PCRs

we believe it can be the most reasonable for the application of Stop \& Go ABR; however, the reader might think that we restricted our analysis to such scenario because in more complex environments Stop \& Go ABR provides poor performance. In order to show that this is not the case, we also provide a glimpse to the performance of Stop \& Go ABR under different operating conditions.

Fig. 5 presents performance results for Stop \& Go ABR in a scenario with 10 TCP connections in a network that spans $300 \mathrm{~km}$ (i.e., $L_{0}=L_{T i}=L_{R i}=$ $100 \mathrm{~km}$ ). The high-priority background traffic is now obtained by the superposition of $10 \mathrm{On}$-Off sources with burstiness 10 . The On and Off periods are exponentially distributed with average equal to 3000 and 27000 slots, respectively, and the PCR of each connection is equal to the global offered background traffic load. The ABR buffer size is 5,000 cells and $t=1250$ cells, keeping the same ratio to the buffer size as for the previous cases; the buffer size for the high-priority background traffic is increased to 10,000 cells, since for high loads the sum of the background traffic PCR is greater than the link capacity.

From the curves in Fig. 5 we see that the performance of Stop \& Go ABR is still rather good, even if the bandwidth-delay product has been increased by one order of magnitude. By comparing these results with those discussed before, it can be seen that the efficiency of TCP connections deteriorates more 

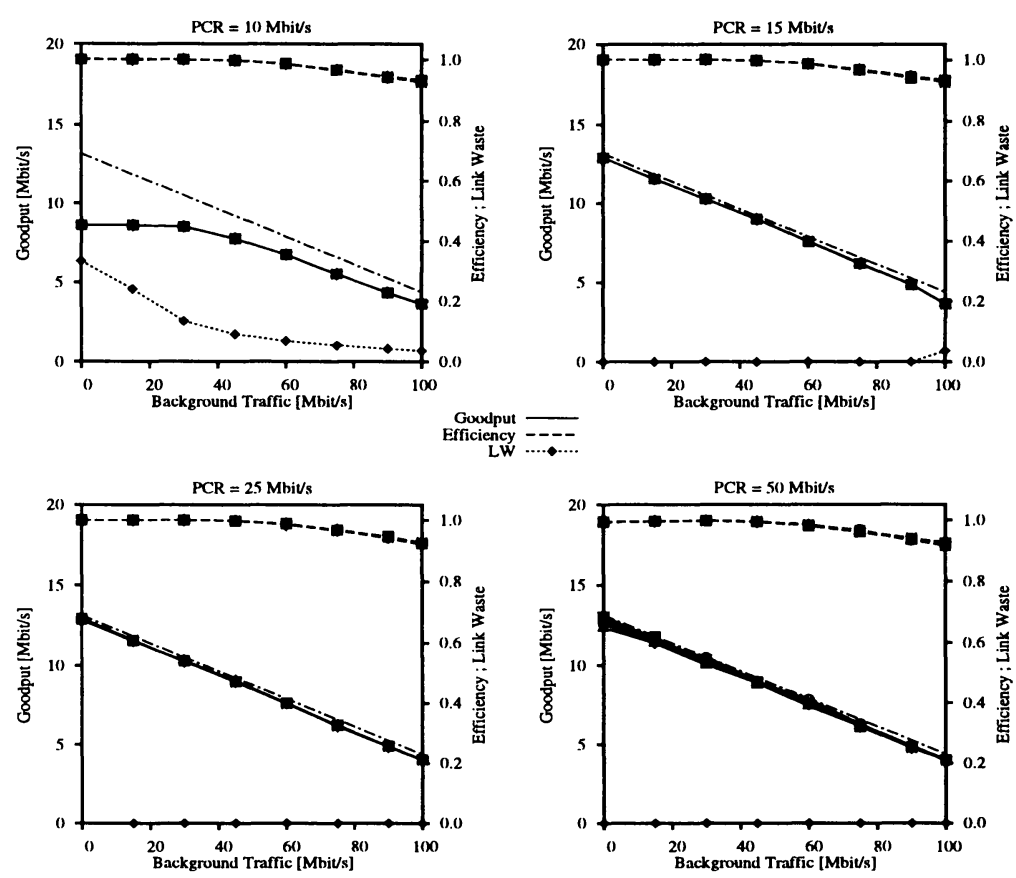

Figure 5 Performance with 10 TCP connections with equal length and PCR

smoothly as the background traffic load increases: this is not due to the larger network span, but to the different characteristics of the background traffic that, being produced by 10 sources, shows a greater variability. It is worthwhile noticing that even in the lower right plot, where the sum of the ABR PCRs is more than 8 times the link capacity, the behavior is still very smooth and fair, and the Stop \& Go algorithm prevents the network from losing any cell of the ABR connections; the same is not true for the background traffic that, even with a 10,000 cell buffer and priority over ABR traffic, suffers from cell losses (not shown in the plots) for loads greater than $60 \mathrm{Mbit} / \mathrm{s}$.

\section{CONCLUSIONS}

In this paper we described the Stop \& Go ABR algorithm, that is a peculiar version of $A B R$ in which sources can transmit only at two different cell rates, called peak cell rate (PCR) and minimum cell rate (MCR).

The performance and fairness of Stop \& Go ABR were evaluated by detailed simulation of a simple ATM LAN configuration with best effort TCP connections as well as interfering VBR connections.

Numerical results showed that Stop \& Go ABR is capable of providing very good performance and fairness for a range of different parameter values.

Rather surprisingly, Stop \& Go ABR proved to perform quite well even in 
the case of network spans of the order of hundreds of $\mathrm{km}$, and very bursty interfering VBR connections.

Further studies on Stop \& Go ABR will consider different switch control algorithms deriving either from RRM ABR or ER ABR.

\section{REFERENCES}

[1] M.Ajmone Marsan, A.Bianco, T.V.Do, L.Jereb, R.Lo Cigno, M.Munafò, "ATM Simulation with CLASS", 'Performance Modeling Tools', Performance Evaluation 24 1995, pp.137-159

[2] M.Ajmone Marsan, A.Bianco, R.Lo Cigno, M.Munafò, "Some Simulation Results about Shaped TCP Connections in ATM Networks", in: D.Kouvatsos (editor), Performance Modeling and Evaluation of ATM Networks - Vol.2, Chapman and Hall, London, 1996

[3] M.Ajmone Marsan, A.Bianco, R.Lo Cigno, M.Munafò, "Four Standard Control Theory Approaches for the Implementation of RRM ABR Services", in: D.Kouvatsos (editor), Performance Modelling and Evaluation of ATM Networks - Vol.3, Chapman and Hall, London, July 1997

[4] M.Ajmone Marsan, A.Bianco, C.Casetti, C.F.Chiasserini, A.Francini, R.Lo cigno, M.Munafò, "An Integrated Simulation Environment for the Analysis of ATM Networks at Multiple Time Scales", to appear on International Journal of Computer Networks and ISDN Systems

[5] ATM Forum AF-TM-0056.000, "ATM Forum Traffic Management Specification", Version 4.0, April 1996

[6] ITU-TSS Study Group 13, Recommendation I.371 "Traffic Control and Congestion Control in B-ISDN", Geneve, Switzerland, July 1995

[7] R.Jain, S.Kalyanaraman, R.Viswanathan, R.Goyal, "A Sample Switch Algorithm", ATM Forum-TM 95-0178R1, February 1995

[8] S.Keung, K.Siu, "Degradation in TCP Performance under Cell Loss", ATM FORUM 94-0490, April 1994

[9] A.Kolarov, G.Ramamurthy, "A Control Theoretic Approach to the Design of Closed Loop Rate Based Control for High Speed ATM Networks", proceedings of IEEE INFOCOM'97, Kobe, Japan, April 1997

[10] S.Mascolo, D.Cavendish, M.Gerla, "ATM Rate Based Congestion Control Using a Smith Predictor: an EPRCA Implementation", proceedings of IEEE INFOCOM'96, S.Francisco, CA, USA, March 1996

[11] H.Li, K.Siu, H.Tzeng, "TCP over ATM with ABR service versus UBR+EPD service", ATM FORUM 95-0718, June 1995

[12] L.Roberts et al. "Enhanced PRCA (Proportional rate-control algorithm), ATM FORUM 94-0735R1, August 1994

[13] A.Romanow, S.Floyd, "Dynamics of TCP Traffic over ATM Networks", IEEE $J S A C$, v.13, no.4, pp.633-641, May 1995

[14] W.R.Stevens, "TCP/IP Illustrated: The Protocols", Vol.1, Addison Wesley, 1994

[15] Y.Zhao, S.Q.Li, S.Sigarto, “A Linear Dynamic Model for Design of Stable Explicit-Rate ABR Control Schemes", proceedings of IEEE INFOCOM'97, Kobe, Japan, April 1997 